\title{
Tunable Polymorphic Transformation Temperature
}

\author{
Masaaki Yokota, Atsushi Ito, Norihito Doki \\ Department of Chemistry and Bioengineering, Iwate University, Morioka, Japan \\ Email: myokota@iwate-u.ac.jp
}

Received 8 September 2015; accepted 20 October 2015; published 23 October 2015

Copyright (C) 2015 by authors and Scientific Research Publishing Inc.

This work is licensed under the Creative Commons Attribution International License (CC BY). http://creativecommons.org/licenses/by/4.0/

c) (i) Open Access

\begin{abstract}
Polymorphic transformation temperature of 2,2':6',2"-Terpyridine (terpy) has been studied. No transformation was observed for unground terpy (orthrhombic form) crystals, while the ground crystals may be transformed into monoclinic form. The transformation temperature was much lower than reported transformation temperature. In addition the transformation temperature decreased with increase of the grinding time. Factors influencing the transformation temperature of terpy were discussed.
\end{abstract}

Keywords

Terpyridine, Crystal, Polymorphic Transformation

\section{Introduction}

A given molecular species has possibility to take more than two crystal structures. This is called as crystal polymorphism. Polymorphism is one of the major research interests in the field of industrial crystallization. In polymorphism, one crystal structure may transform into another structure. This polymorphic transformation occurs via liquid phase and/or in solid state. Recently, solid state transformation has been applied for functional materials such as phase memory and e-ink and this study focus on solid state polymorphic transformation.

2,2':6',2”-Terpyridine (denoted as terpy hereafter, see Figure 1 for molecular structure) is a typical example that has different crystal structures with different emission properties. Orthorhombic $\left(\mathrm{mp}=86^{\circ} \mathrm{C}-88^{\circ} \mathrm{C}\right)$ and monoclinic $\left(\mathrm{mp}=91^{\circ} \mathrm{C}\right.$ ) forms of terpy were reported as shown in Figure 2 [1]. In them, molecular packing manner is different as shown in Figure 2. In addition, molecular geometries are slightly different between them. On heating, the orthorhombic form transforms into the monoclinic form in crystalline state at around $84^{\circ} \mathrm{C}$. In this paper, we show strange transition temperature behavior of terpy that cannot be explained by knowledge of traditional crystallography. 


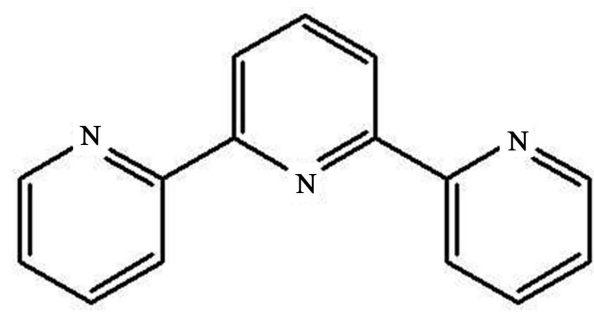

Figure 1. Molecular structure of terpy.

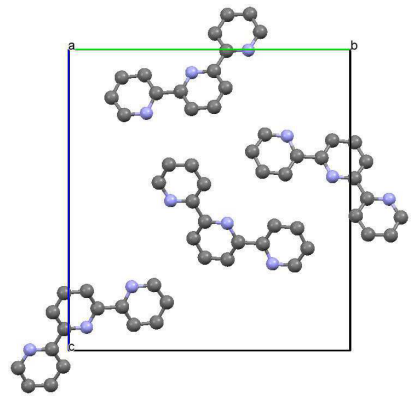

(a)

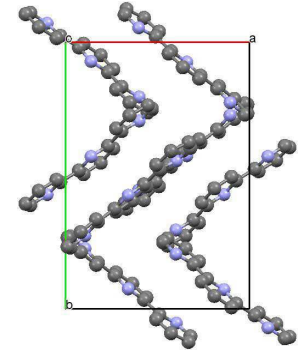

(b)

Figure 2. Two crystal structures of terpy drawn using single crystal structure data [2] [3]: (a) orthrhombic form, (b) monoclinic form.

\section{Experimental Section}

\subsection{Material}

Terpy (MW $=233.27,>98.0 \%$ ) was purchased from Tokyo Kasei and was purified by column chromatography followed by recrystallization using toluene/hexane $(1: 1 \mathrm{vol} / \mathrm{vol})$ mixed solvent. Structure of thus prepared crystals was evaluated as orthorhombic form and was used for following polymorphic transformation experiment.

\subsection{Polymorphic Transformation Experiment}

The starting terpy crystals, orthorhombic form, were ground for a given time manually using a mortar and pestle. The pre-determined grinding time is $0-90 \mathrm{~min}$. About $10 \mathrm{mg}$ of the sample powder was placed in an aluminum pan and differential scanning calorimetry (DSC) analysis was conducted on an EXSTER X-DSC7000 (Hitachi High-tech Science) under nitrogen atmosphere with a heating rate of $5^{\circ} \mathrm{C} / \mathrm{min}$. Solid state transition temperature and melting temperature were determined by the intersection method. Powder XRD patterns of the crystals, sampled before and after a transition peak observed in the DSC, were measured by RINT2200 (Rigaku CuK $\alpha, \lambda$ $=1.5418 \AA$ ). Structure of the crystals was determined by comparing the measured XRD patterns with theoretical XRD patterns (orthorhombic and monoclinic) calculated using reported crystal structure data [2] [3].

\section{Results and Discussion}

\subsection{Influence of Grinding on the Transformation Temperature}

Figure 3 shows DSC profiles of terpy crystals, initially orthorhombic form, as a function of grinding time. Unground terpy crystals (see Figure 3(a)) reveal only one large endothermic peak at around $87^{\circ} \mathrm{C}$, showing melting of the orthorhombic terpy crystals at this temperature. In this case, no transformation from orthorhombic to monoclinic form was occurred. However, when the orthorhombic terpy crystals were ground for a given time, the peak patterns were drastically changed. For example, terpy crystals ground for 1 min (b) showed a broad and weak endothermic peak at $75.4^{\circ} \mathrm{C}$ (estimated by intersection point method). This peak must be indicative of transformation from orthorhombic to monoclinic form. For confirmation, let us check the structures of the crystals sampled before and after the temperature $\left(75.4^{\circ} \mathrm{C}\right)$ by XRD. For XRD measurement of polymorphic substances, care must be taken for temperature change of crystal structures. Our XRD equipment does not have 


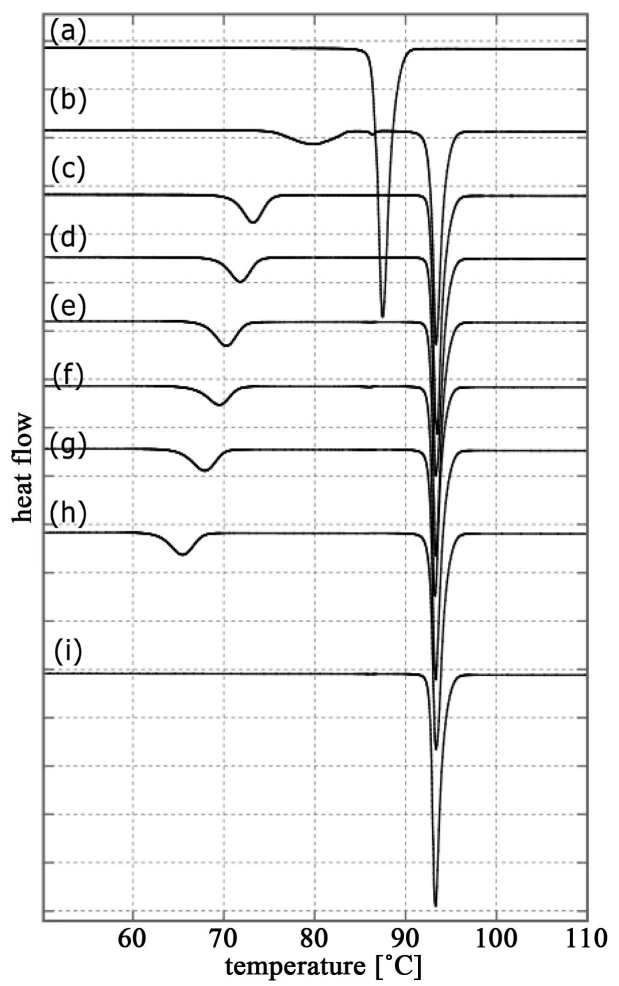

Figure 3. Effect of grinding time on DSC patterns of terpy crystals. (a) Unground; (b) 1 min ground; (c) 10 min ground; (d) 20 min ground; (e) 30 min ground; (f) 40 min ground; (g) 50 min ground; (h) 60 min ground; (i) monoclinic form unground crystals.

temperature control unit and only measurement at room temperature is allowed. Since the high temperature phase (monoclinic in this case) has possibility to transform back into the lower phase (orthorhombic). Thus care must be taken especially when the higher temperature polymorph are measured at lower temperature. For terpy, fortunately, monoclinic form (high temperature phase) do no transformed into orthorhombic form just by cooling. Therefore room temperature XRD measurement is appropriate for evaluation of temperature change of structure of terpy crystals. Figure 4(a) and Figure 4(b) show typical XRD patterns of crystals sampled before (a) and after (b) the transformation temperature $\left(75.4^{\circ} \mathrm{C}\right)$. It may be clearly seen that XRD pattern (a) is agreed with orthorhombic form (d), while that of (b) agreed with monoclinic (c). From this experimental results, the small DSC peak appeared before the melting temperature may be concluded as transformation from orthorhombic to monoclinic form. It is interesting in Figure 3, the transformation temperature shift to lower temperature with increase of the grinding time. It is clear that the transformation temperature may be correlated with grinding time as plotted as Figure 5.

\subsection{Other Factors Affecting the Transformation Temperature}

As shown in Figure 3 and Figure 5, the transformation temperature shifted to lower temperature with increase of grinding time. There are some possibilities to explain this result. One is crystal size effect. Crystal size distribution of the ground terpy crystals were measured and number averaged size of the ground crystals were calculated. The results are plotted against transformation temperature in Figure 6 (the numeric value in bracket refer to grinding time). In Figure 6, data were taken from another set of experiments with the same transformation procedure. Thus the transformation temperature in Figure 6 disagreed with that of Figure 5 . At a glance, the results of Figure 6 looks simple, however, some interesting points may be found. For larger size crystals, e.g. larger than $5.7 \mu \mathrm{m}$, it must be clearly said that size of the crystals influences on transition temperature. In literature, size effect has been explained as surface effect [4] [5]. In case of nano-level particles, surface effect may be understand. However, our case is micrometer level. In such a large scale, surface energy effect is not so sensitive 


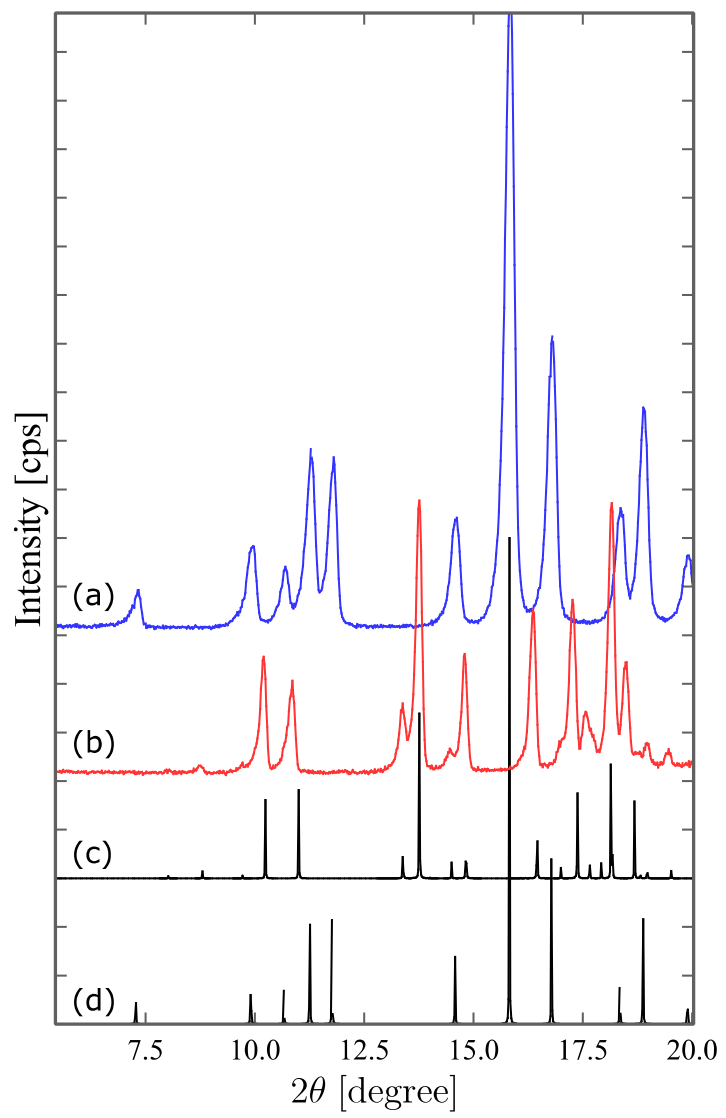

Figure 4. Powder XRD patterns of terpy crystals before (a) and after (b) transformation temperature. For comparison calculated XRD patterns of (c) monoclinic and (d) orthrhombic forms are shown.

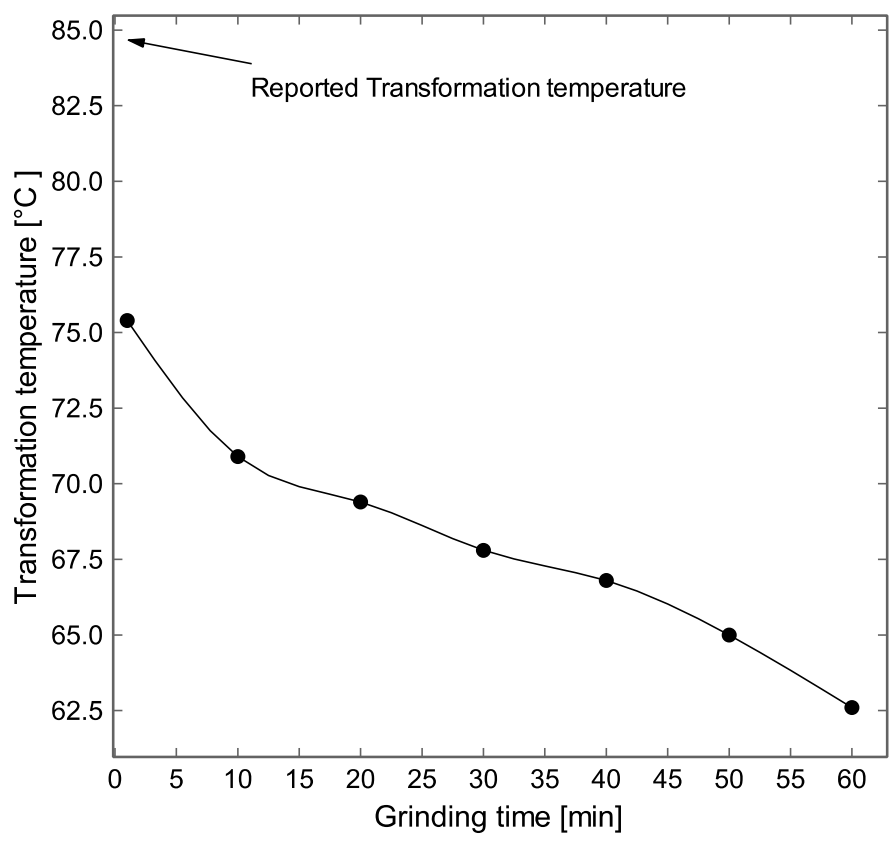

Figure 5. Grinding time dependency of transformation temperature of terpy. 
to crystal size. Thus, the size effect found in this study for crystals larger than $5.7 \mu \mathrm{m}$ must be caused by heat transfer.

Interesting point is found in smaller size region. In smaller size region smaller than $5.7 \mu \mathrm{m}$, increase of grinding time is less effective for particle size. However, the transformation temperature decreased with increasing grinding time. In this region, size effect may not be applied. Another factors must be existed. For another possibility, changes in crystallinity caused by grinding must be considered. As well-known, grinding of particles leads to formation of amorphous. Figure 7 shows typical XRD patterns for terpy crystals with different three

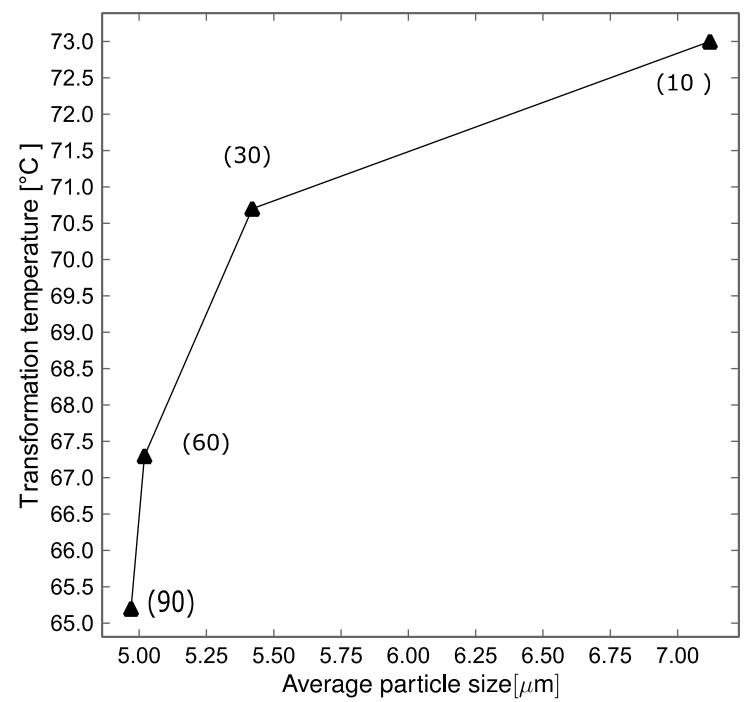

Figure 6. Relation between transformation temperature and average crystal size of terpy.

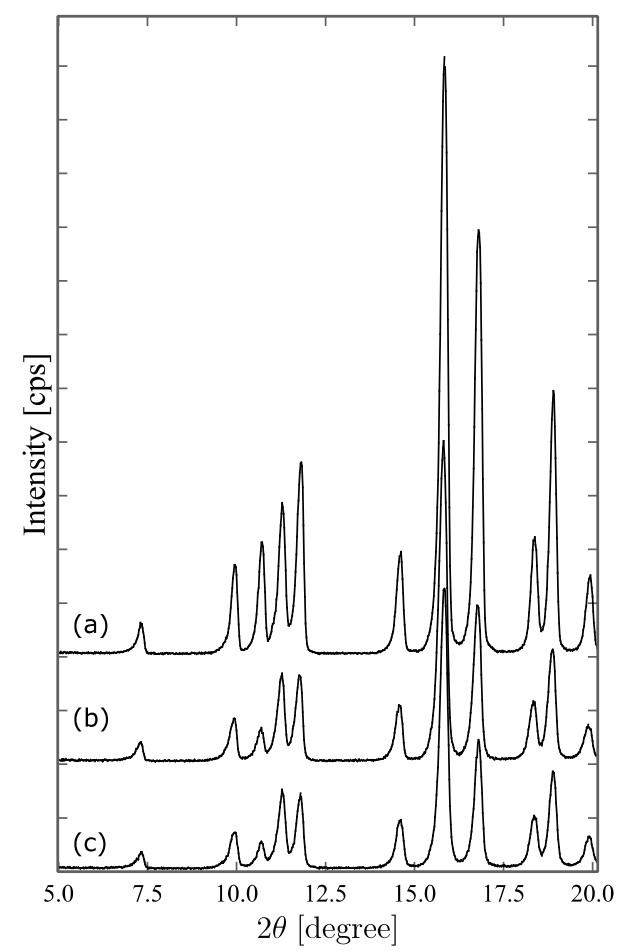

Figure 7. Powder XRD patterns of terpy crystals with different grinding time. (a) 5 min ground; (b) 30 min ground; (c) 60 min ground. 
grinding time. Comparing the three patterns, no apparent difference is found in width of any peak. This suggests us that crystallinity difference is not occurred among the crystals.

Finally, two possibilities are remains. One is introduction of internal stress and/or defect that is believed to influence the transformation temperature. The other possibility is polymorphic transformation caused by grinding. In our previous study, we show existence of small amount of seed crystals of monoclinic form accelerate the transformation of orthorhombic form. Which factor is correct? To answer the question, we need more detailed examination. Anyway, small amount of internal stress and/or another polymorph that can not be detected by XRD measurement must influence the bulk transformation temperature. These results suggest us possibility of tuning a polymorphic transformation temperature without a help of additives.

\section{Conclusion}

Transformation temperature of terpy from orthorhombic to monoclinic form has been studied experimentally. The transformation temperature was found tunable depending on grinding time of the starting orthorhombic crystals. At this moment, the cause of the grinding time dependent transformation temperature is not clear, since obvious changes in crystalline state caused by the grinding are undetected experimentally. This suggests us undetectable small amount of change in crystalline state triggered the polymorphic transformation.

\section{References}

[1] Mutai, T., Satou, H. and Araki, K. (2005) Reproducible On-Off Switching of Solid-State Luminescence by Controlling Molecular Packing through Heat-Mode Interconversion. Nature Materials, 4, 685-687. http://dx.doi.org/10.1038/nmat1454

[2] Bessel, C.A., See, R.F., Jameson, D.L., Churchill, M. and Takeuchi, K.J. (1992) Structural Considerations of Terdentate Ligands: Crystal Structures of 2,2':6',2'-Terpyridine and 2,6-Bis(pyrazol-1-yl)pyridine. Journal of the Chemical Society, Dalton Transactions, 22, 3223-3228. http://dx.doi.org/10.1039/dt9920003223

[3] Bowes, K.F., Clark, I.P., Cole, J.M., Gourlay, M., Griffin, A.M.E., Mahon, M.F., Ooi, L., Parker, A.W., Raithby, P.R., Sparkes, H.A. and Towrie, M. (2005) A New Polymorph of Terpyridine: Variable Temperature X-Ray Diffraction Studies and Solid State Photophysical Properties. CrystEngComm, 7, 269-275. http://dx.doi.org/10.1039/b502275d

[4] Baraldi, C., Gamberini, M.C., Tinti, A., Palazzoli, F. and Ferioli, V. (2009) Vibrational Study of Acetazolamide Polymorphism. Journal of Molecular Structure, 918, 88-96. http://dx.doi.org/10.1016/j.molstruc.2008.07.014

[5] Kanata, T., Yoshikawa, T. and Kubota, K. (1987) Grain-Size Effects on Dielectric Phase Transition of BaTiO $\mathrm{Beram}_{3}$ ics. Solid State Communications, 62, 765-767. http://dx.doi.org/10.1016/0038-1098(87)90044-5 